\title{
CHARACTERIZATIONS OF REAL HYPERSURFACES OF TYPE A IN A COMPLEX SPACE FORM
}

\author{
U-Hang Ki, In-Bae Kim, and Dong Ho Lim
}

\begin{abstract}
Let $M$ be a real hypersurface with almost contact metric structure $(\phi, g, \xi, \eta)$ in a complex space form $M_{n}(c), c \neq 0$. In this paper we prove that if $R_{\xi} \mathcal{L}_{\xi} g=0$ holds on $M$, then $M$ is a Hopf hypersurface in $M_{n}(c)$, where $R_{\xi}$ and $\mathcal{L}_{\xi}$ denote the structure Jacobi operator and the operator of the Lie derivative with respect to the structure vector field $\xi$ respectively. We characterize such Hopf hypersurfaces of $M_{n}(c)$.
\end{abstract}

\section{Introduction}

A complex $n$-dimensional Kaeherian manifold of constant holomorphic sectional curvature $c$ is called a complex space form, which is denoted by $M_{n}(c)$. As is well-known, a complete and simply connected complex space form is complex analytically isometric to a complex projective space $P_{n}(\mathbb{C})$, a complex Euclidean space $\mathbb{C}^{n}$ or a complex hyperbolic space $H_{n}(\mathbb{C})$, according to $c>0$, $c=0$ or $c<0$.

In this paper we consider a real hypersurface $M$ in a complex space form $M_{n}(c), c \neq 0$. Then $M$ has an almost contact metric structure $(\phi, g, \xi, \eta)$ induced from the Kaehler metric and complex structure $J$ on $M_{n}(c)$. The structure vector field $\xi$ is said to be principal if $A \xi=\alpha \xi$ is satisfied, where $A$ is the shape operator of $M$ and $\alpha=\eta(A \xi)$. In this case, it is known that $\alpha$ is locally constant ([3]) and that $M$ is called a Hopf hypersurface.

Typical examples of Hopf hypersurfaces in $P_{n}(\mathbb{C})$ are homogeneous ones, namely those real hypersurfaces are given as orbits under subgroup of the projective unitary group $P U(n+1)$. Takagi [8] completely classified such hypersurfaces as six model spaces which are said to be $A_{1}, A_{2}, B, C, D$ and $E$. On the other hand, real hypersurfaces in $H_{n}(\mathbb{C})$ have been investigated by Berndt [1], Montiel and Romero [4] and so on. Berndt [1] classified all homogeneous Hopf hyersurfaces in $H_{n}(\mathbb{C})$ as four model spaces which are said to be $A_{0}, A_{1}$, $A_{2}$ and $B$.

Received April 2, 2008.

2000 Mathematics Subject Classification. Primary 53C40; Secondary 53C15.

Key words and phrases. real hypersurface, structure Jacobi operator, Hopf hypersurface.

This work was supported by the research fund of Hankuk University of Foreign Studies. 
We introduce the following theorems without proof due to Okumura [6] for $c>0$, and Montiel and Romero [4] for $c<0$ respectively.

Theorem O-MR ([4], [6]). Let $M$ be a real hypersurface of $M_{n}(c), c \neq 0$. It satisfies $A \phi-\phi A=0$ on $M$ if and only if $M$ is locally congruent to one of the following hypersurfaces:

(1) In cases $P_{n}(\mathbb{C})$,

$\left(A_{1}\right)$ a tube of radius $r$ over a hyperplane $P_{n-1}(\mathbb{C})$, where $0<r<\frac{\pi}{2}, r \neq \frac{\pi}{4}$,

$\left(A_{2}\right)$ a tube of radius $r$ over a totally geodesic $P_{k}(\mathbb{C})(1 \leq k \leq n-2)$, where $0<r<\frac{\pi}{2}, r \neq \frac{\pi}{4}$.

(2) In cases $H_{n}(\mathbb{C})$,

$\left(A_{0}\right)$ a horosphere in $H_{n}(\mathbb{C})$, that is, a Montiel tube,

$\left(A_{1}\right)$ a tube of a totally geodesic hyperplane $H_{k}(\mathbb{C})(k=1$ or $n-1)$,

$\left(A_{2}\right)$ a tube of a totally geodesic $H_{k}(\mathbb{C})(1 \leq k \leq n-2)$.

Let $M$ be a real hypersurface of type $\left(A_{1}\right)$ or $\left(A_{2}\right)$ in $P_{n}(\mathbb{C})$ or type $\left(A_{0}\right)$, $\left(A_{1}\right)$ or $\left(A_{2}\right)$ in $H_{n}(\mathbb{C})$. Then $M$ is said to be of type $(A)$ for simplicity.

The curvature tensor field $R$ on a Riemannian manifold $(M, g)$ is defined by

$$
R(X, Y)=\left[\nabla_{X}, \nabla_{Y}\right]-\nabla_{[X, Y]}
$$

for any vector fields $X$ and $Y$ on $(M, g)$. We define the Jacobi operator $R_{X}$ by $R_{X}=R(\cdot, X) X$ with respect to a unit vector field $X$. Then we see that $R_{X}$ is self-adjoint endomorphism of the tangent space. It is related with (the Jacobi vector equation) $\nabla_{\dot{\gamma}}\left(\nabla_{\dot{\gamma}} Y\right)+R(Y, \dot{\gamma}) \dot{\gamma}=0$ along a geodesic $\gamma$ on $M$, where $\dot{\gamma}$ denotes the velocity vector field of $\gamma$.

When we study a real hypersurface $M$ in a complex space form, we will call the Jacobi operator $R_{\xi}$ with respect to the structure vector field $\xi$ a structure Jacobi operator on the real hypersurface $M$. Recently it is known that there are no real hypersurfaces in $M_{n}(c)$ with parallel structure Jacobi operator $R_{\xi}$ (see [7]). Some works have also studied several conditions on the structure Jacobi operator $R_{\xi}$ and given some results on the classification of real hypersurfaces of type $(A)$ in $M_{n}(c)([2],[4],[5]$ and [6] etc).

The induced operator on a real hypersurface $M$ from the 2 -form $\mathcal{L}_{\xi} g$ will be denoted by the same symbol, that is, $\left(\mathcal{L}_{\xi} g\right)(X, Y)=g\left(\left(\mathcal{L}_{\xi} g\right) X, Y\right)$ for any vector fields $X$ and $Y$ on $M$, where $\mathcal{L}_{\xi}$ denotes the operator of the Lie derivative with respect to the structure vector field $\xi$. In this paper we shall study a real hypersurface in a non-flat complex space form $M_{n}(c)$ which satisfies $R_{\xi} \mathcal{L}_{\xi} g=0$. We give another characterization of real hypersurface of type $(A)$ in $M_{n}(c)$ by the above condition. The main purpose of the present paper is to establish Theorem 5.1.

All manifolds in the present paper are assumed to be connected and of class $C^{\infty}$ and the real hypersurfaces supposed to be orientable. 


\section{Preliminaries}

Let $M$ be a real hypersurface immersed in a complex space form $M_{n}(c)$, and $N$ be a unit normal vector field of $M$. By $\widetilde{\nabla}$ we denote the Levi-Civita connection with respect to the Fubini-Study metric tensor $\widetilde{g}$ of $M_{n}(c)$. Then the Gauss and Weingarten formulas are given respectively by

$$
\widetilde{\nabla}_{X} Y=\nabla_{X} Y+g(A X, Y) N, \quad \widetilde{\nabla}_{X} N=-A X
$$

for any vector fields $X$ and $Y$ on $M$, where $g$ denotes the Riemannian metric tensor of $M$ induced from $\widetilde{g}$, and $A$ is the shape operator of $M$ in $M_{n}(c)$. For any vector field $X$ on $M$ we put

$$
J X=\phi X+\eta(X) N, \quad J N=-\xi,
$$

where $J$ is the almost complex structure of $M_{n}(c)$. Then we see that $M$ induces an almost contact metric structure $(\phi, g, \xi, \eta)$, that is,

$$
\begin{aligned}
& \phi^{2} X=-X+\eta(X) \xi, \quad \phi \xi=0, \quad \eta(\xi)=1, \\
& g(\phi X, \phi Y)=g(X, Y)-\eta(X) \eta(Y), \quad \eta(X)=g(X, \xi)
\end{aligned}
$$

for any vector fields $X$ and $Y$ on $M$.

Since the almost complex structure $J$ is parallel, we can verify from the Gauss and Weingarten formulas the followings:

$$
\begin{gathered}
\nabla_{X} \xi=\phi A X \\
\left(\nabla_{X} \phi\right) Y=\eta(Y) A X-g(A X, Y) \xi .
\end{gathered}
$$

Since the ambient manifold is of constant holomorphic sectional curvature $c$, we have the following Gauss and Codazzi equations respectively:

$$
\begin{gathered}
R(X, Y) Z=\frac{c}{4}\{g(Y, Z) X-g(X, Z) Y+g(\phi Y, Z) \phi X-g(\phi X, Z) \phi Y \\
-2 g(\phi X, Y) \phi Z\}+g(A Y, Z) A X-g(A X, Z) A Y \\
\left(\nabla_{X} A\right) Y-\left(\nabla_{Y} A\right) X=\frac{c}{4}\{\eta(X) \phi Y-\eta(Y) \phi X-2 g(\phi X, Y) \xi\}
\end{gathered}
$$

for any vector fields $X, Y$ and $Z$ on $M$, where $R$ denotes the Riemannian curvature tensor of $M$.

From the Gauss equation (2.3) the structure Jacobi operator $R_{\xi}$ is given by

$$
R_{\xi} X=R(X, \xi) \xi=\frac{c}{4}\{X-\eta(X) \xi\}+\alpha A X-\eta(A X) A \xi
$$

for any vector field $X$ on $M$.

Let $W$ be a unit vector field on $M$ with the same direction of the vector field $-\phi \nabla_{\xi} \xi$, and let $\mu$ be the length of the vector field $-\phi \nabla_{\xi} \xi$ if it does not vanish, and zero (constant function) if it vanishes. Then it is easily seen from (2.1) that

$$
A \xi=\alpha \xi+\mu W
$$


where $\alpha=\eta(A \xi)$. We notice here that $W$ is orthogonal to $\xi$. We put

$$
\Omega=\{p \in M \mid \mu(p) \neq 0\} .
$$

Then $\Omega$ is an open subset of $M$. If we put $X=W$ into (2.5) and make use of (2.6), then we have on $\Omega$

$$
R_{\xi} W=-\alpha \mu \xi+\left(\frac{c}{4}-\mu^{2}\right) W+\alpha A W .
$$

In what follows we assume that $\Omega \neq \emptyset$, that is, the structure vector field $\xi$ is not principal, and we discuss our arguments on $\Omega$ unless otherwise stated.

\section{Real hypersurfaces satisfying $R_{\xi} \mathcal{L}_{\xi} g=0$}

Let $M$ be a real hypersurface in a complex space form $M_{n}(c), c \neq 0$, satisfying $R_{\xi} \mathcal{L}_{\xi} g=0$. This condition together with (2.1) implies that

$$
R_{\xi}(\phi A-A \phi)=0
$$

or equivalently

$$
(\phi A-A \phi) R_{\xi}=0 .
$$

If we apply $\xi$ to (3.1) and make use of (2.5) and (2.6), then it is easy to see that $\alpha \neq 0$ and hence

$$
A \phi W=-\frac{c}{4 \alpha} \phi W
$$

on $\Omega$. Applying $W$ to (3.1) and taking account of (2.5), (2.6) and (3.3), we have

$$
\alpha A \phi A W+\frac{c}{4} \phi A W=0 .
$$

The application of $W$ to (3.2) gives rise to

$$
\alpha^{2} A \phi A W-\alpha^{2} \phi A^{2} W-\alpha\left(\frac{c}{4}-\mu^{2}\right) \phi A W-\left(\frac{c^{2}}{16}-\frac{c}{4} \mu^{2}-\alpha^{2} \mu^{2}\right) \phi W=0
$$

by virtue of (2.6), (2.7) and (3.3). From the above two equations, we get

$$
\begin{aligned}
& \alpha^{2} A^{2} W+\alpha\left(\frac{c}{2}-\mu^{2}\right) A W-\alpha \mu\left(\frac{c}{2}+\alpha \gamma+\alpha^{2}-\mu^{2}\right) \xi \\
& +\left(\frac{c^{2}}{16}-\frac{c}{4} \mu^{2}-\alpha^{2} \mu^{2}\right) W=0,
\end{aligned}
$$

where we have put

$$
\gamma=g(A W, W) .
$$

Applying $\phi W$ to (3.1) and using (2.5), (2.6), (2.7) and (3.3), we have

$$
\begin{aligned}
& \alpha^{2} A^{2} W+\frac{c}{2} \alpha A W-\alpha \mu\left(\frac{c}{2}+\alpha \gamma+\alpha^{2}\right) \xi \\
& +\left(\frac{c^{2}}{16}-\frac{c}{4} \mu^{2}-\alpha^{2} \mu^{2}-\alpha \gamma \mu^{2}\right) W=0 .
\end{aligned}
$$

It is easily seen from (3.4) and (3.5) that

$$
A W=\mu \xi+\gamma W
$$


on $\Omega$. If we substitute (3.6) into (3.5) and make use of (2.6) and (3.6), then we obtain

$$
\left(\frac{c}{4}+\alpha \gamma\right)\left(\frac{c}{4}+\alpha \gamma-\mu^{2}\right)=0 .
$$

Applying $\phi X$ to (3.1) and using (2.5), (2.6), (3.3) and (3.6), we can verify that

$$
\begin{aligned}
& \alpha^{2} A \phi A \phi X+\frac{c}{4} \alpha \phi A \phi X+\alpha^{2} A^{2} X+\frac{c}{4} \alpha A X \\
= & \alpha\left\{\alpha\left(\frac{c}{4}+\alpha^{2}+\mu^{2}\right) \eta(X)+\mu\left(\frac{c}{2}+\alpha \gamma+\alpha^{2}\right) w(X)\right\} \xi \\
& +\mu\left(\frac{c}{4}+\alpha \gamma+\alpha^{2}\right)\{\alpha \eta(X)+\mu w(X)\} W
\end{aligned}
$$

for any vector field $X$ on $M$, where the 1 -form $w$ is the dual one of $W$, that is,

$$
w(X)=g(W, X) .
$$

Differentiating the smooth function $\mu=g(A \xi, W)$ along any vector field $X$ on $M$ and using (2.1), (2.4), (2.6), (3.3) and (3.6), we have

$$
X \mu=g\left(\left(\nabla_{\xi} A\right) W+\frac{c}{4 \alpha} \gamma \phi W, X\right) .
$$

Since we have $\left(\nabla_{\xi} A\right) W=\nabla_{\xi}(\mu \xi+\gamma W)-A \nabla_{\xi} W$, we see from the above equation that the gradient vector field $\nabla \mu$ of $\mu$ is given by

$$
\nabla \mu=-(A-\gamma I) \nabla_{\xi} W+(\xi \mu) \xi+(\xi \gamma) W+\left(\mu^{2}+\frac{c}{4 \alpha} \gamma\right) \phi W
$$

where $I$ indicates the identity transformation on $M$. If we differentiate $\alpha=$ $g(A \xi, \xi)$ along any vector field $X$ and take account of $(2.1),(2.4),(2.6),(3.3)$ and (3.6), then we obtain $\nabla \alpha=\left(\nabla_{\xi} A\right) \xi+\frac{c}{2 \alpha} \mu \phi W$ and hence

$$
\nabla \alpha=\mu \nabla_{\xi} W+(\xi \alpha) \xi+(\xi \mu) W+\mu\left(\frac{3 c}{4 \alpha}+\alpha\right) \phi W .
$$

As a similar argument as the above, we can see that the gradient vector fields of $\gamma=g(A W, W)$ and $-\frac{c}{4 \alpha}=g(A \phi W, \phi W)$ are given respectively by

$$
\nabla \gamma=-(A-\gamma I) \nabla_{W} W+(W \mu) \xi+(W \gamma) W+\mu\left(\gamma-\frac{c}{2 \alpha}\right) \phi W
$$

and

$$
\frac{c}{4 \alpha} \nabla \alpha=-\alpha\left(A+\frac{c}{4 \alpha} I\right) \phi \nabla_{\phi W} W+\frac{c}{4 \alpha}((\phi W) \alpha) \phi W .
$$

Taking inner product of (3.12) with $\xi$ and $W$ and using (2.6) and (3.6), we obtain

$$
4 \alpha^{2} \mu g\left(\nabla_{\phi W} W, \phi W\right)=c \xi \alpha, \quad \alpha(4 \alpha \gamma+c) g\left(\nabla_{\phi W} W, \phi W\right)=c W \alpha
$$

respectively. The above two relations imply that

$$
\alpha \mu W \alpha=\left(\frac{c}{4}+\alpha \gamma\right) \xi \alpha
$$


By means of (2.1), (2.2), (2.6), (3.3) and (3.6), we can verify that

$$
\begin{aligned}
\left(\nabla_{\phi W} A\right) \xi & =\nabla_{\phi W} A \xi-A \nabla_{\phi W} \xi \\
& =\mu \nabla_{\phi W} W+\left\{(\phi W) \alpha-\frac{c}{4 \alpha} \mu\right\} \xi+\left\{(\phi W) \mu+\frac{c}{4}-\frac{c}{4 \alpha} \gamma\right\} W
\end{aligned}
$$

and

$$
\left(\nabla_{\xi} A\right) \phi W=-\left(A+\frac{c}{4 \alpha} I\right) \phi \nabla_{\xi} W+\mu\left(\frac{c}{4 \alpha}+\alpha\right) \xi+\mu^{2} W+\frac{c}{4 \alpha^{2}}(\xi \alpha) \phi W .
$$

Therefore it follows from the equation (2.4) of Codazzi that

$$
\begin{aligned}
& \mu \nabla_{\phi W} W+\left(A+\frac{c}{4 \alpha} I\right) \phi \nabla_{\xi} W \\
= & -\left\{(\phi W) \alpha-\mu\left(\frac{c}{2 \alpha}+\alpha\right)\right\} \xi-\left\{(\phi W) \mu-\mu^{2}-\frac{c}{4 \alpha} \gamma\right\} W+\frac{c}{4 \alpha^{2}}(\xi \alpha) \phi W .
\end{aligned}
$$

We can also verify from $\left(\nabla_{\xi} A\right) W-\left(\nabla_{W} A\right) \xi$ that

$$
\begin{aligned}
& \mu \nabla_{W} W+(A-\gamma I) \nabla_{\xi} W \\
= & (\xi \mu-W \alpha) \xi+(\xi \gamma-W \mu) W+\left(\mu^{2}-\frac{c}{4}-\alpha \gamma-\frac{c}{4 \alpha} \gamma\right) \phi W .
\end{aligned}
$$

Taking inner product of this equation with $\xi$ and $W$, we find

$$
\xi \mu=W \alpha \quad \text { and } \quad \xi \gamma=W \mu
$$

respectively, and hence the initial equation is reduced to

$$
\mu \nabla_{W} W+(A-\gamma I) \nabla_{\xi} W=\left(\mu^{2}-\frac{c}{4}-\alpha \gamma-\frac{c}{4 \alpha} \gamma\right) \phi W
$$

As a similar argument as the above, it follows from $\left(\nabla_{W} A\right) \phi W-\left(\nabla_{\phi W} A\right) W$ that

$$
\begin{aligned}
& (A-\gamma I) \nabla_{\phi W} W-\left(A+\frac{c}{4 \alpha} I\right) \phi \nabla_{W} W \\
= & \left\{(\phi W) \mu-\frac{c}{2}-\alpha \gamma-\frac{c}{4 \alpha} \gamma\right\} \xi+\left\{(\phi W) \gamma-\mu \gamma+\frac{c}{4 \alpha} \mu\right\} W-\frac{c}{4 \alpha^{2}}(W \alpha) \phi W .
\end{aligned}
$$

If we eliminate the term $(A-\gamma I) \nabla_{\xi} W$ from (3.9) and (3.16), then we obtain

$$
\mu \nabla_{W} W=\nabla \mu-(\xi \mu) \xi-(\xi \gamma) W-\left(\frac{c}{4}+\alpha \gamma+\frac{c}{2 \alpha} \gamma\right) \phi W
$$

Substituting (3.18) into (3.11) and making use of (2.6), (3.3), (3.6) and (3.15), we get

$$
\begin{aligned}
& (A-\gamma I) \nabla \mu+\mu \nabla \gamma \\
= & \{(\alpha-\gamma) \xi \mu+2 \mu \xi \gamma\} \xi+\mu(\xi \mu+W \gamma) W \\
& +\left\{\mu^{2}\left(\gamma-\frac{c}{2 \alpha}\right)-\left(\frac{c}{4 \alpha}+\gamma\right)\left(\frac{c}{4}+\alpha \gamma+\frac{c}{2 \alpha} \gamma\right)\right\} \phi W .
\end{aligned}
$$


If we compare (3.9) with (3.10), then we can find

$$
\begin{aligned}
& (A-\gamma I) \nabla \alpha+\mu \nabla \mu \\
= & \{(\alpha-\gamma) \xi \alpha+2 \mu \xi \mu\} \xi+\mu(\xi \alpha+\xi \gamma) W \\
& +\mu\left\{\mu^{2}+\frac{c}{4 \alpha} \gamma-\left(\frac{c}{4 \alpha}+\gamma\right)\left(\frac{3 c}{4 \alpha}+\alpha\right)\right\} \phi W
\end{aligned}
$$

by eliminating $\nabla_{\xi} W$ in both (3.9) and (3.10).

If we eliminate the term $\nabla_{\phi W} W$ from (3.12) and (3.14), and make use of (2.6), (3.3) and (3.6), then we obtain

$$
\begin{aligned}
\frac{c}{4 \alpha^{2}} \mu \nabla \alpha= & \left(A \phi+\frac{c}{4 \alpha} \phi\right)^{2} \nabla_{\xi} W+\frac{c}{4 \alpha^{2}} \mu(\xi \alpha) \xi \\
& +\frac{c}{4 \alpha^{2}}\left(\frac{c}{4 \alpha}+\gamma\right)(\xi \alpha) W+\frac{c}{4 \alpha^{2}} \mu((\phi W) \alpha) \phi W
\end{aligned}
$$

Since $\phi^{2}=-I+\eta \otimes \xi$ and $\eta\left(\nabla_{\xi} W\right)=0$, it is easily seen that

$$
\left(A \phi+\frac{c}{4 \alpha} \phi\right)^{2} \nabla_{\xi} W=\frac{1}{\alpha}\left\{\alpha A \phi A \phi+\frac{c}{4} \phi A \phi-\frac{c}{4}\left(A+\frac{c}{4 \alpha} I\right)\right\} \nabla_{\xi} W .
$$

Putting $X=\nabla_{\xi} W$ into (3.8), it is easy to see that

$$
\left(\alpha A \phi A \phi+\frac{c}{4} \phi A \phi\right) \nabla_{\xi} W=-\left(\alpha A^{2}+\frac{c}{4} A\right) \nabla_{\xi} W .
$$

From the above results we have $\left(A \phi+\frac{c}{4 \alpha} \phi\right)^{2} \nabla_{\xi} W=-\left(A+\frac{c}{4 \alpha} I\right)^{2} \nabla_{\xi} W$, and hence the initial equation is given by

$$
\begin{aligned}
\frac{c}{4 \alpha^{2}} \mu \nabla \alpha= & -\left(A+\frac{c}{4 \alpha} I\right)^{2} \nabla_{\xi} W+\frac{c}{4 \alpha^{2}} \mu(\xi \alpha) \xi \\
& +\frac{c}{4 \alpha^{2}}\left(\frac{c}{4 \alpha}+\gamma\right)(\xi \alpha) W+\frac{c}{4 \alpha^{2}} \mu((\phi W) \alpha) \phi W .
\end{aligned}
$$

Finally, if we eliminate the term $\nabla_{\xi} W$ from (3.10) and the last equation, and take account of (2.6), (3.3) and (3.6), then we obtain

$$
\begin{aligned}
& \left\{\alpha^{2} A^{2}+\frac{c}{2} \alpha A+\frac{c}{4}\left(\frac{c}{4}+\mu^{2}\right) I\right\} \nabla \alpha \\
= & \left\{\left[\alpha^{2}\left(\frac{c}{2}+\alpha^{2}+\mu^{2}\right)+\frac{c}{4}\left(\frac{c}{4}+\mu^{2}\right)\right] \xi \alpha+\alpha \mu\left(\frac{c}{2}+\alpha \gamma+\alpha^{2}\right) \xi \mu\right\} \xi \\
& +\left\{\mu\left[\alpha\left(\frac{c}{2}+\alpha \gamma+\alpha^{2}\right)+\frac{c}{4}\left(\frac{c}{4 \alpha}+\gamma\right)\right] \xi \alpha+\left[\alpha^{2}\left(\mu^{2}+\gamma^{2}\right)+\frac{c}{2} \alpha \gamma+\frac{c^{2}}{16}\right] \xi \mu\right\} W \\
& +\frac{c}{4} \mu^{2}((\phi W) \alpha) \phi W .
\end{aligned}
$$

\section{Some lemmas}

Let $M$ be a real hypersurface satisfying $R_{\xi} \mathcal{L}_{\xi} g=0$ in a complex space form $M_{n}(c), c \neq 0$. In this section we assume that $\Omega \neq \emptyset$, and we shall prove some lemmas, which will be used later. 
Lemma 4.1. If $\frac{c}{4}+\alpha \gamma=\mu^{2}$ holds on a non-empty open subset $\Omega_{0}$ of $\Omega$, then we have

$$
\alpha \nabla \alpha=(\xi \alpha) A \xi-3 \mu\left(\alpha^{2}-\frac{c}{4}\right) \phi W
$$

on $\Omega_{0}$.

Proof. It follows from (3.13) and the hypothesis that

$$
\mu \xi \alpha=\alpha W \alpha
$$

on $\Omega_{0}$. Since we have $\alpha \nabla \gamma+\gamma \nabla \alpha=2 \mu \nabla \mu$, it is easily seen from (3.15) and (4.2) that

$$
\alpha^{2} W \mu=\left(\mu^{2}+\frac{c}{4}\right) \xi \alpha .
$$

If we substitute $2 \mu \nabla \mu=\alpha \nabla \gamma+\gamma \nabla \alpha$ into (3.19) and make use of the hypothesis, then we have

$$
\begin{aligned}
& \left\{\alpha A+\left(\frac{c}{4}+\mu^{2}\right) I\right\} \nabla \gamma+\gamma(A-\gamma I) \nabla \alpha \\
= & 2 \mu\{(\alpha-\gamma) \xi \mu+2 \mu \xi \gamma\} \xi+2 \mu^{2}(\xi \mu+W \gamma) W-\frac{c}{2 \alpha^{2}} \mu^{3}(3 \alpha+2 \gamma) \phi W .
\end{aligned}
$$

Taking inner product of (4.4) with $\phi W$ and using (3.3), we obtain

$$
\alpha(\phi W) \gamma-\gamma(\phi W) \alpha=-\frac{c}{2 \alpha} \mu(3 \alpha+2 \gamma) .
$$

If we substitute $\alpha \nabla \gamma+\gamma \nabla \alpha=2 \mu \nabla \mu$ into (3.20), then we have

$$
\begin{aligned}
& (2 A-\gamma I) \nabla \alpha+\alpha \nabla \gamma \\
= & 2\{(\alpha-\gamma) \xi \alpha+2 \mu \xi \mu\} \xi+2 \mu(\xi \alpha+\xi \gamma) W-\frac{c}{\alpha^{2}} \mu\left(\mu^{2}+\frac{c}{8}\right) \phi W .
\end{aligned}
$$

Taking inner product of (4.6) with $\phi W$ and using (3.3), we get

$$
\alpha\left(\mu^{2}+\frac{c}{4}\right)(\phi W) \alpha-\alpha^{3}(\phi W) \gamma=c \mu\left(\mu^{2}+\frac{c}{8}\right) .
$$

It follows from (4.5) and (4.7) that

$$
\alpha(\phi W) \alpha=-3 \mu\left(\alpha^{2}-\frac{c}{4}\right) .
$$

From (2.6), (3.3) and (3.6), we see that the subspace spanned by the three vectors $\xi, W$ and $\phi W$ is invariant under the shape operator $A$. Thus eliminating the gradient vector field $\nabla \gamma$ from (4.4) and (4.6), we can find

$$
\left\{\alpha^{2} A^{2}+\frac{c}{2} \alpha A-\frac{c}{4}\left(\mu^{2}-\frac{c}{4}\right) I\right\} \nabla \alpha=x \xi+y W+z \phi W
$$

where $x, y$ and $z$ are smooth functions on $\Omega_{0}$. If we compare (3.21) with the above relation, then it is easy to see that the gradient vector field $\nabla \alpha$ of $\alpha$ is expressed by a linear combination of $\xi, W$ and $\phi W$ only. Therefore, using (2.6), (4.2) and (4.8), we have (4.1). 
Lemma 4.2. If $\frac{c}{4}+\alpha \gamma=0$ holds on a non-empty open subset of $\Omega$, then we have

$$
\nabla \alpha=(\xi \alpha) \xi-3 \alpha \mu \phi W
$$

on the open subset.

Proof. It follows from (3.13) and (3.15) that $W \alpha=0$ and $\xi \mu=0$. From the hypothesis, $W \alpha=0$ gives rise to $W \gamma=0$ on the open subset. We see from (3.3) that $A \phi W=\gamma \phi W$.

Using the equation $\alpha \nabla \gamma+\gamma \nabla \alpha=0$ and the above results, the equations (3.19) and (3.20) are rewritten as

$$
\begin{aligned}
& \alpha(A-\gamma I) \nabla \mu-\mu \gamma \nabla \alpha=-2 \mu \gamma(\xi \alpha) \xi-\frac{3}{4} c \mu^{2} \phi W, \\
& (A-\gamma I)^{2} \nabla \alpha+\mu(A-\gamma I) \nabla \mu \\
= & \left\{\left(\alpha^{2}+\mu^{2}+\gamma^{2}+\frac{c}{2}\right) \xi \alpha+\mu^{2} \xi \gamma\right\} \xi+\mu(\alpha-\gamma)(\xi \alpha) W
\end{aligned}
$$

respectively. If we eliminate the term $(A-\gamma I) \nabla \mu$ from (4.10) and (4.11), then we obtain

$$
\begin{aligned}
& \left\{\alpha^{2} A^{2}+\frac{c}{2} \alpha A+\frac{c}{4}\left(\frac{c}{4}-\mu^{2}\right) I\right\} \nabla \alpha \\
= & \left\{\alpha^{2}\left(\alpha^{2}+\mu^{2}+\frac{c}{2}\right)+\frac{c}{4}\left(\frac{c}{4}-\mu^{2}\right)\right\}(\xi \alpha) \xi+\alpha \mu\left(\alpha^{2}+\frac{c}{4}\right)(\xi \alpha) W+\frac{3}{4} c \alpha \mu^{3} \phi W,
\end{aligned}
$$

where we have used $\alpha \nabla \gamma+\gamma \nabla \alpha=0$. Comparing (3.21) with (4.12) and using $\xi \mu=0$, we get

$$
\nabla \alpha=(\xi \alpha) \xi+\frac{1}{2}\{(\phi W) \alpha-3 \alpha \mu\} \phi W,
$$

from which $(\phi W) \alpha=-3 \alpha \mu$. Thus we have (4.9).

Lemma 4.3. We have $\xi \alpha=\xi \mu=\xi \gamma=0$ and $W \alpha=W \mu=W \gamma=0$ on $\Omega$.

Proof. First of all, we assume that there is a point $p$ of $\Omega$ such that $(W \alpha)(p) \neq$ 0 . Then there is an open neighborhood $\Omega_{1}$ of $p$ in $\Omega$ such that $\xi \alpha \neq 0$ and $\frac{c}{4}+\alpha \gamma \neq 0$ on $\Omega_{1}$ by (3.13). This means that

$$
\frac{c}{4}+\alpha \gamma=\mu^{2}
$$

holds on $\Omega_{1}$ by (3.7). Therefore, from Lemma 4.1 and in the proof of this lemma, we see that the equations (4.1), (4.2), (4.3) and (4.8) are satisfied on $\Omega_{1}$.

If we compare (4.1) with (3.10) and take account of (3.15) and (4.2), then we obtain

$$
\nabla_{\xi} W=-4 \alpha \phi W
$$


Moreover, substituting (4.13) into (3.14) and using (3.6) and (4.8), we get

$$
\mu \nabla_{\phi W} W=-\frac{c}{4 \alpha} \mu \xi+\frac{c}{4 \alpha^{2}}(\xi \alpha) \phi W
$$

Let $v$ be the dual 1-form of the unit vector field $\phi W$, that is,

$$
v(X)=g(\phi W, X)
$$

for any vector field $X$ on $M$. Then it follows from (4.1) that

$$
X \alpha^{2}=2(\xi \alpha) \eta(A X)-6 \mu\left(\alpha^{2}-\frac{c}{4}\right) v(X) .
$$

Since we have $[X, Y] \alpha^{2}=X Y \alpha^{2}-Y X \alpha^{2}$, we can verify from (4.15) that

$$
\begin{aligned}
& (X \xi \alpha) \eta(A Y)-(Y \xi \alpha) \eta(A X)+2(\xi \alpha) g(A \phi A X, Y)-\frac{c}{2}(\xi \alpha) g(\phi X, Y) \\
& -3\left(\alpha^{2}-\frac{c}{4}\right)\{(X \mu) v(Y)-(Y \mu) v(X)\}-6 \alpha \mu\{(X \alpha) v(Y)-(Y \alpha) v(X)\} \\
& -6 \mu\left(\alpha^{2}-\frac{c}{4}\right) d v(X, Y)=0
\end{aligned}
$$

by virtue of the equations (2.1) and (2.4), where

$$
2 d v(X, Y)=X v(Y)-Y v(X)-v([X, Y])
$$

for any vector fields $X$ and $Y$ on $M$. Since we have $\alpha \xi \mu=\mu \xi \alpha$ by (3.15) and (4.2), and $d v(\xi, \phi W)=0$ by (2.1) and (3.3), putting $X=\phi W$ and $Y=\xi$ into (4.16) yields

$$
(\phi W)(\xi \alpha)=\mu\left(\frac{c}{4 \alpha^{2}}-9\right) \xi \alpha .
$$

If we put $X=\phi W$ and $Y=W$ into (4.16) and make use of (4.1), (4.2), (4.3), (4.14) and (4.17), then we obtain

$$
(\xi \alpha)\left(\alpha^{2}-\frac{c}{4}\right)=0
$$

on $\Omega_{1}$. Since $\xi \alpha \neq 0$ by (3.13) and our assumption, this result shows that $\alpha$ is a constant on $\Omega_{1}$, and a contradiction.

Thus we have $W \alpha=0$ on the whole $\Omega$. Since $W \alpha=0$ on $\Omega$, we have $\xi \mu=0$ by $(3.15)$ and

$$
\left(\frac{c}{4}+\alpha \gamma\right) \xi \alpha=0
$$

on $\Omega$ by (3.13).

Next we assume that there is a non-empty open subset $\Omega_{2}$ of $\Omega$ such that $\xi \alpha \neq 0$ on $\Omega_{2}$. Then we have

$$
\frac{c}{4}+\alpha \gamma=0
$$


and hence (4.9) holds on $\Omega_{2}$ by Lemma 4.2. If we make use of the relation $[X, Y] \alpha=X Y \alpha-Y X \alpha$, then it is easy to verify from (4.9) that

$$
\begin{aligned}
& (X \xi \alpha) \eta(Y)-(Y \xi \alpha) \eta(X)+(\xi \alpha) g((\phi A+A \phi) X, Y) \\
& -3 \mu\{(X \alpha) v(Y)-(Y \alpha) v(X)\}-3 \alpha\{(X \mu) v(Y)-(Y \mu) v(X)\} \\
& -6 \alpha \mu d v(X, Y)=0
\end{aligned}
$$

for any vector fields $X$ and $Y$ on $M$. Since we see that $W \alpha=0, A \phi W=\gamma \phi W$ by (3.3), $2 d v(W, \phi W)=g\left(\nabla_{\phi W} W, \phi W\right)$ and $\alpha W \mu=-\gamma \xi \alpha$ by (3.15) and $\alpha \xi \gamma+\gamma \xi \alpha=0$, putting $X=W$ and $Y=\phi W$ into the above equation yields

$$
5 \gamma \xi \alpha=3 \alpha \mu g\left(\nabla_{\phi W} W, \phi W\right) .
$$

If we take inner product of (3.12) with $\xi$ and make use of (2.6) and $\frac{c}{4 \alpha}=-\gamma$, then we obtain

$$
\gamma \xi \alpha=-\alpha \mu g\left(\nabla_{\phi W} W, \phi W\right) .
$$

Combining (4.18) with (4.19), we have $\gamma \xi \alpha=0$ on $\Omega_{2}$ and a contradiction.

Therefore $\xi \alpha=0$ on the whole $\Omega$. It follows from (3.7) that

$$
\mu\left(\frac{c}{2}+\alpha \gamma\right) \nabla \mu=\left(\frac{c}{2}+\alpha \gamma-\mu^{2}\right)(\gamma \nabla \alpha+\alpha \nabla \gamma) .
$$

Since $\xi \alpha=0$ and $\xi \mu=0$ on $\Omega$, the above equation shows that $\xi \gamma=0$. Since $\xi \gamma=0$ on $\Omega$, we have $W \mu=0$ by (3.15). Together with $W \alpha=0$, the above equation also gives rise to $W \gamma=0$ on $\Omega$.

Lemma 4.4. If it satisfies $d v(\xi, X)=0$ for any vector field $X$ on $\Omega$, then we have $\Omega=\emptyset$.

Proof. By use of (2.2), (2.6) and (3.6), it is easily seen from $v(X)=g(\phi W, X)$ that $d v(\xi, X)=0$ is equivalent to

$$
\nabla_{\xi} W=\gamma \phi W
$$

If we compare (3.16) with (4.20) and take account of (3.3), then we obtain

$$
\mu \nabla_{W} W=\left(\mu^{2}+\gamma^{2}-\frac{c}{4}-\alpha \gamma\right) \phi W .
$$

Assume that there is a point $p$ in $\Omega$ such that $\frac{c}{4}+\alpha \gamma \neq 0$ at $p$. Then it follows from (3.7) that

$$
\frac{c}{4}+\alpha \gamma=\mu^{2}
$$

on an open neighborhood of $p$. By Lemmas 4.1 and 4.3 , we have

$$
\alpha \nabla \alpha=-3 \mu\left(\alpha^{2}-\frac{c}{4}\right) \phi W
$$

on the open neighborhood. Substituting (4.20) into (3.9) and making use of (4.22) and Lemma 4.3, we obtain

$$
\alpha \nabla \alpha=\mu\left(\alpha^{2}+\mu^{2}+\frac{c}{2}\right) \phi W,
$$


from which together with (4.23),

$$
4 \alpha^{2}+\mu^{2}=\frac{c}{4}
$$

It follows from (4.22) and (4.24) that

$$
4 \alpha+\gamma=0
$$

If we substitute (4.21) into (3.11) and taking account of (3.3), (4.22), (4.24), (4.25) and Lemma 4.3, then we get

$$
\alpha \nabla \gamma=\mu\left(12 \alpha^{2}-\frac{c}{2}\right) \phi W
$$

Since we have $4 \nabla \alpha+\nabla \gamma=0$ from (4.25), we can verify that $c=0$ from (4.23) and (4.26), and a contradiction.

Thus we have $\frac{c}{4}+\alpha \gamma=0$ on the whole $\Omega$. By Lemmas 4.2 and 4.3 , we have

$$
\nabla \alpha=-3 \alpha \mu \phi W
$$

Substituting (4.20) into (3.10) and using Lemma 4.3, we obtain

$$
\alpha \nabla \alpha=\mu\left(\alpha^{2}+\frac{c}{2}\right) .
$$

It follows from (4.27) and (4.28) that $\alpha^{2}+\frac{c}{8}=0$ and hence $\nabla \alpha=0$. Thus from (4.27) we have $\alpha \mu=0$ on $\Omega$ and hence a contradiction.

Lemma 4.5. If there is a smooth function $f$ on $\Omega$ such that

$$
f \phi W=f_{1} \nabla \alpha+f_{2} \nabla \mu+f_{3} \nabla \gamma,
$$

then $f$ vanishes identically on $\Omega$, where $f_{1}, f_{2}$ and $f_{3}$ are the polynomials with respect to $\alpha, \mu$ and $\gamma$ respectively.

Proof. Taking inner product of (4.29) with any vector field $X$ on $M$, we have $f v(X)=f_{1} X \alpha+f_{2} X \mu+f_{3} X \gamma$. If we differentiate this equation along any vector field $\mathrm{Y}$ on $M$ and take the skew-symmetric parts in $X$ and $Y$, then we obtain

$$
\begin{aligned}
& (Y f) v(X)-(X f) v(Y)-2 f d v(X, Y) \\
= & \left(Y f_{1}\right) X \alpha-\left(X f_{1}\right) Y \alpha+\left(Y f_{2}\right) X \mu-\left(X f_{2}\right) Y \mu+\left(Y f_{3}\right) X \gamma-\left(X f_{3}\right) Y \gamma
\end{aligned}
$$

on $\Omega$. Putting $Y=\xi$ into the above equation and using Lemma 4.3, we get

$$
(\xi f) v(X)+2 f d v(\xi, X)=0 .
$$

Since $v(\phi W)=1$ and $d v(\xi, \phi W)=0$ by (2.1) and (3.3), we see from the above equation that $\xi f=0$ and hence $f d v(\xi, X)=0$ for any vector field $X$. Thus we have $f=0$ on $\Omega$ by Lemma 4.4 . 


\section{Proof of theorems}

In this section, we shall prove the following theorems.

Theorem 5.1. Let $M$ be a real hypersurface satisfying $R_{\xi} \mathcal{L}_{\xi} g=0$ in a complex space form $M_{n}(c), c \neq 0$. Then $M$ is a Hopf hypersurface in $M_{n}(c)$.

Proof. We assume that $\Omega=\{p \in M \mid \mu(p) \neq 0\}$ is not empty. Then, by (3.7), we see that there is a non-empty open subset of $\Omega$ such that either $\frac{c}{4}+\alpha \gamma=\mu^{2}$ or $\frac{c}{4}+\alpha \gamma=0$ on the open subset.

In the case where $\frac{c}{4}+\alpha \gamma=0$, it follows from Lemmas 4.2 and 4.3 that

$$
\nabla \alpha=-3 \alpha \mu \phi W \text {. }
$$

By Lemma 4.5, we see that $\alpha \mu=0$ and hence it is a contradiction.

Thus we have $\frac{c}{4}+\alpha \gamma=\mu^{2}$ on the whole $\Omega$. By Lemmas 4.1 and 4.3 , the gradient vector field $\nabla \alpha$ of $\alpha$ is given by

$$
\alpha \nabla \alpha=-3 \mu\left(\alpha^{2}-\frac{c}{4}\right) \phi W
$$

By Lemma 4.5, the above equation implies that

$$
\alpha^{2}=\frac{c}{4}
$$

on $\Omega$. Since $\nabla \alpha=0$ by (5.1), it follows from (3.20) and Lemma 4.3 that

$$
\nabla \mu=\left\{\mu^{2}+\frac{c}{4 \alpha} \gamma-\left(\frac{c}{4 \alpha}+\gamma\right)\left(\frac{3 c}{4 \alpha}+\alpha\right)\right\} \phi W .
$$

From the above equation and Lemma 4.5 , it is easy to see that $\mu$ is a constant and

$$
\mu^{2}=-\frac{c}{8}
$$

on $\Omega$ by virtue of $\frac{c}{4}+\alpha \gamma=\mu^{2}$. Since (5.1) and (5.2) give a contradiction, the set $\Omega$ must be empty. Thus $M$ is a Hopf hypersurface.

Theorem 5.2. Let $M$ be a real hypersurface in a complex space form $M_{n}(c)$, $c \neq 0$. Then it satisfies $R_{\xi} \mathcal{L}_{\xi} g=0$ on $M$ if and only if $M$ is locally congruent to one of the model spaces of type $(A)$.

Proof. Let $M$ satisfies $R_{\xi} \mathcal{L}_{\xi} g=0$. Then $M$ is a Hopf hypersurface by Theorem 5.1, that is, $A \xi=\alpha \xi$. Therefore our assumption $R_{\xi}(\phi A-A \phi)=0$ or equivalently $(\phi A-A \phi) R_{\xi}=0$ are given by

$$
\begin{aligned}
& \alpha A \phi A-\alpha A^{2} \phi+\frac{c}{4}(\phi A-A \phi)=0, \\
& \alpha A \phi A-\alpha \phi A^{2}-\frac{c}{4}(\phi A-A \phi)=0 .
\end{aligned}
$$

On the other hand, if we differentiate $A \xi=\alpha \xi$ covariantly and make use of the equation $(2,4)$ of Codazzi, then we have

$$
A \phi A-\frac{\alpha}{2}(\phi A+A \phi)-\frac{c}{4} \phi=0 .
$$


Let $X$ be any vector field on $M$ such that $A X=\lambda X$. Then it follows from (5.4) that

$$
\left(\alpha \lambda+\frac{c}{4}\right) A \phi X=\lambda\left(\alpha \lambda+\frac{c}{4}\right) \phi X .
$$

From (5.5) we also obtain

$$
\left(\lambda-\frac{\alpha}{2}\right) A \phi X=\frac{1}{2}\left(\alpha \lambda+\frac{c}{2}\right) \phi X .
$$

Assume that there is a point $p$ of $M$ such that $\alpha \lambda+\frac{c}{4}=0$ at $p$. Then we see from (5.7) that $\lambda-\frac{\alpha}{2} \neq 0$, and $A \phi X=\frac{c}{4(2 \lambda-\alpha)} \phi X$ at $p$. Applying $X$ to (5.3) and using $\alpha \lambda+\frac{c}{4}=0$, we obtain $\lambda=0$ and hence $c=0$ at $p$. It is a contradiction.

Therefore we see that $\alpha \lambda+\frac{c}{4} \neq 0$ on $M$, and from (5.6) that $A \phi X=\lambda \phi X$ for any vector field $X$ satisfying $A X=\lambda X$. Therefore from this results we obtain

$$
\phi A=A \phi
$$

on the whole $M$.

Conversely if it satisfies (5.8), then it is easily seen that (5.3) holds, that is, $R_{\xi} \mathcal{L}_{\xi} g=0$ is satisfies on $M$. Thus Theorem 5.2 follows from Theorem $\mathrm{O}-\mathrm{MR}$.

\section{References}

[1] J. Berndt, Real hypersurfaces with constant principal curvatures in complex hyperbolic space, J. Reine Angew. Math. 395 (1989), 132-141.

[2] J. T. Cho and U.-H. Ki, Real hypersurfaces of a complex projective space in terms of the Jacobi operators, Acta Math. Hungar. 80 (1998), no. 1-2, 155-167.

[3] U.-H. Ki and Y. J. Suh, On real hypersurfaces of a complex space form, Math. J. Okayama Univ. 32 (1990), 207-221.

[4] S. Montiel and A. Romero, On some real hypersurfaces of a complex hyperbolic space, Geom. Dedicata 20 (1986), no. 2, 245-261.

[5] R. Niebergall and P. J. Ryan, Real hypersurfaces in complex space forms, Tight and taut submanifolds (Berkeley, CA, 1994), 233-305, Math. Sci. Res. Inst. Publ., 32, Cambridge Univ. Press, Cambridge, 1997.

[6] M. Okumura, On some real hypersurfaces of a complex projective space, Trans. Amer. Math. Soc. 212 (1975), 355-364.

[7] M. Ortega, J. D. Pérez, and F. G. Santos, Non-existence of real hypersurfaces with parallel structure Jacobi operator in nonflat complex space forms, Rocky Mountain J. Math. 36 (2006), no. 5, 1603-1613.

[8] R. Takagi, On homogeneous real hypersurfaces in a complex projective space, Osaka J. Math. 10 (1973), 495-506.

U-HANG KI

The National Academy of Science

SEOUl 137-044, KoreA

E-mail address: uhangki2005@yahoo.co.kr 
IN-BAE KIM

DePartment of Mathematics

HANkUk University of Foreign Studies

SEOUl 130-791, KoreA

E-mail address: ibkim@hufs.ac.kr

DONG HO LIM

Department of Mathematics

Hankuk University of Foreign Studies

SEOUl 130-791, KoreA

E-mail address: dhlnys@hufs.ac.kr 